\title{
Telemedicine System Based on Medical Consultation Assistance Integration
}

\author{
Jhe-Wei Lin'1, Cheng-Yan Siao', Rong-Guey Chang1, Mei-Ling Hsu² \\ ${ }^{1}$ Department of Computer Science and Information Engineering, Advanced Institute of Manufacturing with High-Tech \\ Innovations, National Chung Cheng University, Chiayi \\ ${ }^{2}$ Department of Information Technology, Ditmanson Medical Foundation Chia-Yi Christian Hospital, Chiayi \\ Email: hcyen103m@cs.ccu.edu.tw
}

How to cite this paper: Lin, J.-W., Siao, C.-Y., Chang, R.-G. and Hsu, M.-L. (2021) Telemedicine System Based on Medical Consultation Assistance Integration. Journal of Software Engineering and Applications, 14, 537-548.

https://doi.org/10.4236/jsea.2021.1410031

Received: August 16, 2021

Accepted: October 5, 2021

Published: October 8, 2021

Copyright (c) 2021 by author(s) and Scientific Research Publishing Inc. This work is licensed under the Creative Commons Attribution International License (CC BY 4.0).

http://creativecommons.org/licenses/by/4.0/

\begin{abstract}
With the aging of the global population, how to provide effective telemedicine for the aging population has become a very important issue, especially for the elderly with limited mobility. If there is a complete telemedicine system, it will not only greatly improve medical efficiency. It can reduce the chance of contact between people and avoid the medical risks caused by severe special infectious pneumonia. This paper focuses on the development of a highefficiency telemedicine system platform that conforms to international standard data exchange formats. This system platform can not only solve the problem of shortage of medical staff but also allow patients to be free from medical outpatient time constraints. Achieve the effect of telemedicine at any time, and digitize the medical process rules to establish a complete online telemedicine system platform.
\end{abstract}

\section{Keywords}

Telemedicine, Medical Care System, Fast Healthcare Interoperability Resources

\section{Introduction}

With the development of medical technology, the average life expectancy of the global population continues to increase [1]. How to improve the efficiency of social medical treatment has become a topic that cannot be ignored. According to research by the United Nations Department of Economic and Social Affairs Office [2], the global support ratio of the elderly population by 2050, the number will be reduced to 4 , indicating that the country's burden of supporting the elderly population is increasing. If there is no comprehensive medical system, when 
an unexpected outbreak occurs, the national medical system will break.

With the continuous development of current technology, new types of viruses have gradually affected the world. Particularly serious in recent years, COVID-19 is very heavy impact. [3] It not only affects global economic development but also affects the human lifestyle. The current medical system can no longer cope with the medical capacity of countries around the world, if there is a set of instant telemedicine systems that can quickly transmit medical information without delay when patients are consulting, it not only can reduce the amount of medical treatment, but also make it more convenient for the elderly with mobility impairments, and for hospitals, it can reduce the chance of infection caused by crowd gathering.

Before the emergence of COVID-19, many countries have actively promoted telemedicine systems. Portugal proposed the National Strategic Telehealth Plan [4] for medical infrastructure, collaboration systems, and services to improve the regulatory framework. In addition, Germany proposed the Telemedical Maritime Assistance Service [5] to ensure that when a global emergency medical situation occurs, the medical hotline immediately operates radio medical treatment. After the outbreak of COVID-19 in 2019, the development of telemedicine systems has been accelerated, and telemedicine systems are gradually moving towards precision, immediacy, and complete research and development. According to the definition of the World Health Organization [6], telemedicine refers to medical behaviors and health information transmission using real-time video and data communication technology for diagnosis, consultation, and treatment. In addition, with the development of Industry 4.0, the new thinking of smart medical treatment is so derived [7], telemedicine is an important pioneer in the development of smart medical systems. It not only brings revolutionary changes to traditional medical services, but doctors can also assist medical decision-making, reduce errors, and avoid medical disputes from rapidly accumulating data. Create new opportunities for smart medical care.

We designed and implemented a web page telemedicine system that allows patients to conduct medical consultations in a remote manner through various information equipments. In addition to regularly feeding back the system platform with data for self-measurement of physiological conditions, it also provides long-term medical staff tracking the patient's physical condition to meet nonessential contact requirements, that is, the system platform exchanges opinions between the two parties. The biggest difference between our system and other systems is that we are designing for the real-time feedback system, which conforms to the international standard data exchange format. Under the premise, we analyze the format of the data. Even if the amount of data is huge, the system can still operate normally, without affecting the rights of patients and influencing doctors to treat patients.

In this paper, we introduce a high-efficiency and real-time telemedicine system platform. The other parts of the paper are described below. Section 2 is the 
related work. Section 3 is the description of the platform operation process. Then we show the experimental results in Section 4. Finally, we conclude this paper in brief in Section 5 .

\section{Related Work}

\subsection{Telemedicine Communication Technology}

With the rise of 5th generation mobile networks [8] and WIFI6 [9], the real-time transmission of data in telemedicine is accelerated, especially in areas with inconvenient transportation. Telemedicine can not only solve the medical inconvenience of people in space, but also shorten the time for people to seek medical treatment.

Telemedicine uses communication technology to lift the traditional medical care fixed area restrictions for patients, thereby improving medical quality and reducing medical costs. Therefore, communication technology is one of the core technologies for the development of traditional medical care into telemedicine. The increase in the penetration rate of wireless communication networks, the improvement of physiological measurement technology, and the rapid development of care medical equipment will drive the rapid growth of the telemedicine industry market.

\subsection{Health Level Seven International}

Health level seven international is an association defined in the telemedicine data exchange format [10]. By formulating a complete electronic health information framework and related standards, the 7 in the name corresponds to the seventh layer of the Open System Interconnection Model. It is expected that this framework, is conducive to the exchange, integration, and sharing of patients' medical information, and improves the privacy of patients and the quality of medical care [11].

\subsection{Fast Healthcare Interoperability Resources}

Fast Healthcare Interoperability Resources(FHIR) is an international medical data exchange standard format [12], which is mainly used to describe the data format and data elements of Electronic Health Records, and provide standard system information in the Application Programming Interface [13], and strengthened patient data exchange, covering not only medical institutions, medical records, hospital stay, and discharge and referral records, but also data exchange between insurance institutions and insurance institutions.

FHIR's biggest aim is to accelerate the effective communication of medical information among medical units and to use medical information widely on computers, tablets, and smartphones so that hospitals and patients can quickly and easily receive medical service information. FHIR is composed of a series of resource implementations. Medical staff use copying and fine-tuning resources to solve medical practice and management problems. The resource is a set of excel 
worksheets that can be organized and used to record data. At present, in the FHIR R4 version of the standard [14], 145 types of resources are summarized. These resources can be divided into five categories, including foundation, base, clinical, financial, and specialized. The fields and fields contained in the resource are listed in a hierarchical directory. The affiliation, the data description of the field is attached next to the field.

In this way, each hospital can develop a customized FHIR extended data format definition, and publish the customized FHIR extended data format on the official website to provide everyone with a unified format standard reference source. Even after the patient is registered, the patient's personal information can be included in the Implementation Guide, and the relevant patient code and files can be attached for other hospitals to download and apply.

\section{The Proposed Approach}

\subsection{Patient Medical Form}

We will refer to the FHIR standard specification to design the form design system, and then exchange information with other medical units and cross-system interconnection functions. In our system, we will design for three FHIR resource types [15], which include Patient, Encounter, and CarePlan, as shown in Figure 1.

In addition, we use RESTful to obtain or access the JSON or XML format text files of the hospital's internal medical record database. As shown in Table 1, various RESTful operation types are written in the form of Application Programming Interface, so that users can use them more efficiently.

\subsection{System Interface}

We use HTML, CSS, and Javascript programming languages to design a web

\begin{tabular}{|c|c|c|c|c|}
\hline 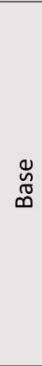 & $\begin{array}{l}\text { Individuals } \\
\text { - Patient } 5 \\
\text { - Practitioner } 3 \\
\text { - PractitionerRole } 2 \\
\text { - RelatedPerson } 2 \\
\text { - Person } 2 \\
\text { - Group } 1\end{array}$ & $\begin{array}{l}\text { Entities } \\
\text { - Organization } 3 \\
\text { - HealthcareService } 2 \\
\text { - Endpoint } 2 \\
\text { - Location } 3 \\
\text { - Substance } 2 \\
\text { - Device } 2 \\
\text { - DeviceComponent } 1 \\
\text { - DeviceMetric } 1\end{array}$ & $\begin{array}{l}\text { Workflow } \\
\text { - Task } 2 \\
\text { - Appointment } 3 \\
\text { - AppointmentResponse } 3 \\
\text { - Schedule } 3 \\
\text { - Slot } 3 \\
\text { - ProcessRequest } 2 \\
\text { - ProcessResponse } 2\end{array}$ & $\begin{array}{l}\quad \text { Management } \\
\text { - Encounter } 2 \\
\text { - EpisodeOfCare } 2 \\
\text { - Flag } 1 \\
\text { - List } 1 \\
\text { - Library } 2\end{array}$ \\
\hline$\frac{\overline{0}}{\frac{0}{\bar{U}}}$ & $\begin{array}{l}\text { Summary } \\
\text { - AllergyIntolerance } 3 \\
\text { - AdverseEvent } 0 \\
\text { - Condition (Problem) } 3 \\
\text { - Procedure } 3 \\
\text { - FamilyMemberHistroy } 2 \\
\text { - Clinicallmpression } 0 \\
\text { - Detectedlssue } 1\end{array}$ & $\begin{array}{l}\quad \text { Diagnostics } \\
\text { - Observation } 5 \\
\text { - DiagnosticReport } 3 \\
\text { - Specimen } 2 \\
\text { - BodySite } 1 \\
\text { - ImagingStudy } 3 \\
\text { - ImagingManifest } 1 \\
\text { - QuestionnairResponse } 3 \\
\text { - Sequence } 1\end{array}$ & $\begin{array}{l}\text { Medications } \\
\text { - MedicationAdministration } 2 \\
\text { - MedicationDispense } 2 \\
\text { - MedicationStatement } 3 \\
\text { - Medication } 3 \\
\text { - Immunization } 3 \\
\text { - Immunization Recommendation } 1\end{array}$ & $\begin{array}{l}\quad \text { Care Provision } \\
\text { - CarePlan } 2 \\
\text { - CareTeam } 2 \\
\text { - Goal } 2 \\
\text { - ReferralRequest } 1 \\
\text { - ProcedureRequest } 3 \\
\text { - NutritionOrder } 2 \\
\text { - VisionPrescription } 1 \\
\text { - RiskAssessment } 1 \\
\text { - RequestGrop } 2\end{array}$ \\
\hline
\end{tabular}

Figure 1. FHIR resource type. 
interface for user login and functional requirements, as shown in Figure 2, and finally, a complete medical interface [16], as shown in Figure 3, so that medical staff can easily view patient's material.

\subsection{Medical Information Browsing Interface}

At present, smartphones have become popular, and many hospitals have introduced

Table 1. RESTFful operation instructions.

\begin{tabular}{|c|c|}
\hline Operation & Method \\
\hline Create & PSOT https://example.com/path/\{resourceType\} \\
\hline Read & GET https://example.com/path/\{resourceType $\} /\{$ id $\}$ \\
\hline Update & PUT https://example.com/path/\{resourceType $\} /\{$ id $\}$ \\
\hline Delete & DELETE https://example.com/path/\{resourceType $\} /\{\mathrm{id}\}$ \\
\hline Search & GET https://example.com/path/\{resourceType\}?search parameters... \\
\hline History & GET https://example.com/path/\{resourceType\}/\{id\} history \\
\hline Transaction & PSOT https://example.com/path/(POST transaction bundle to the system) \\
\hline Operation & GET https://example.com/path/\{resourceType $\} /\{\mathrm{id}\} / \$\{$ opname $\}$ \\
\hline
\end{tabular}

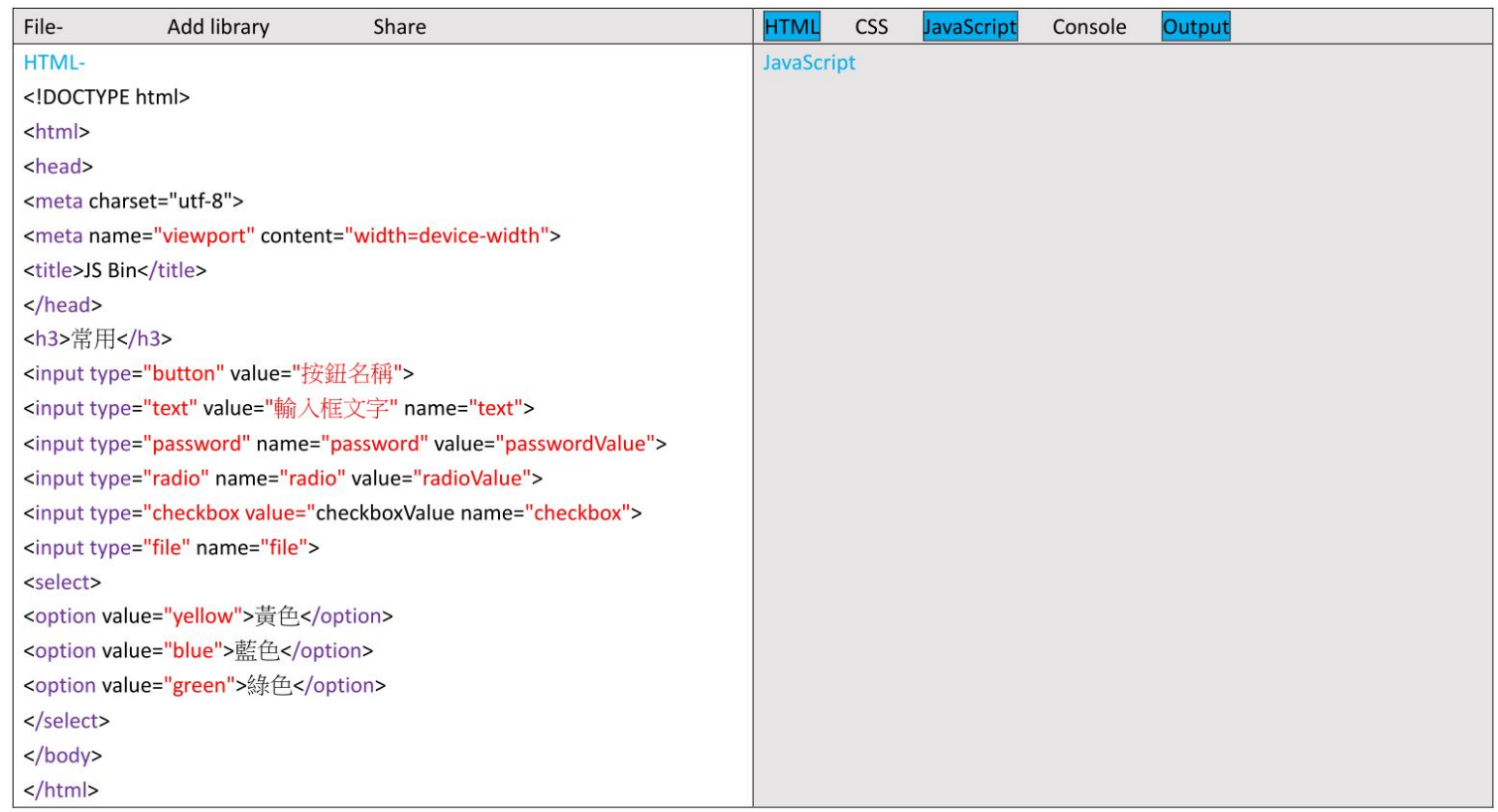

Figure 2. Front-end system design layout example.

\begin{tabular}{|c|c|c|c|c|c|c|c|c|c|c|c|}
\hline $\begin{array}{l}\text { Subject } \\
\text { ID }\end{array}$ & $\begin{array}{l}\text { Name } \\
\text { Latest } \\
\text { IMEI }\end{array}$ & $\begin{array}{c}\text { Active } \\
\text { Monitoring }\end{array}$ & $\begin{array}{l}\text { Last } \\
\text { Survey } \\
\text { Score }\end{array}$ & $\begin{array}{c}\text { Last Survey } \\
\text { Time }\end{array}$ & $\begin{array}{c}\text { Avg KCAL Last } 24 \\
\text { hrs(AVG KCAL when } \\
\text { mounted) }\end{array}$ & $\begin{array}{l}\text { Current Battery } \\
\text { Time Left Estimate }\end{array}$ & Data & $\begin{array}{l}\text { Survey } \\
\text { Answers }\end{array}$ & $\begin{array}{l}\text { Survey } \\
\text { Alarm }\end{array}$ & Message & $\begin{array}{c}\text { Switch Active } \\
\text { Flag }\end{array}$ \\
\hline \multirow[t]{2}{*}{1014} & END & YES & 1 & $2014-06-23$ & $1.325507(1.260188)$ & NA & view & view & set & edit & Deactivate \\
\hline & & & & $21: 26: 02$ & & & & & & & \\
\hline \multirow[t]{2}{*}{1001} & END & NO & 0 & 2013-12-02 & & NA & view & view & set & edit & Activate \\
\hline & & & & $10: 07: 56$ & & & & & & & \\
\hline
\end{tabular}

Figure 3. Example of viewing user data on the medical side. 
tablets to replace computers for medical care. In response to the above mobile communication devices, we will propose a mobile communication device system environment for telemedicine. Various mobile communication devices have different screen sizes. We use responsive web design technology to solve the problem of cross-platform use. As shown in Figure 4, the system can automatically detect the size of the user's Internet device and automatically adjust the graphic content of medical web pages for different screens to give users the best browse screen.

In addition, we integrate the Software Development Kit tools with the web system to build a rich application framework on the Android system and the iOS system. The application framework provided by the system can be adjusted according to the situation and can provide exclusive resources for different device settings. In turn, the efficiency of medical data transmission can be improved.

\section{Experimental Result}

\subsection{Telemedicine System Implementation}

We use Visual Studio as the development tool for the telemedicine system platform, which is mainly compatible with other telemedicine systems and has a complete set of relevant cross-platform device development tools.

\subsection{Medical Records Conversion Electronic}

The primary task of establishing a telemedicine system platform is to convert paper medical records into electronic storage. We use the medical records of Chiayi Christian Hospital as a reference, as shown in Figure 5, and use the database to design an electronic data table that matches it, as shown in Figure 6. When the patient creates the file for the first time, the corresponding data is stored in the FHIR database at the same time.

\subsection{Patient Consultation Process}

The proportion of the aging in the world is gradually increasing, and the proportion

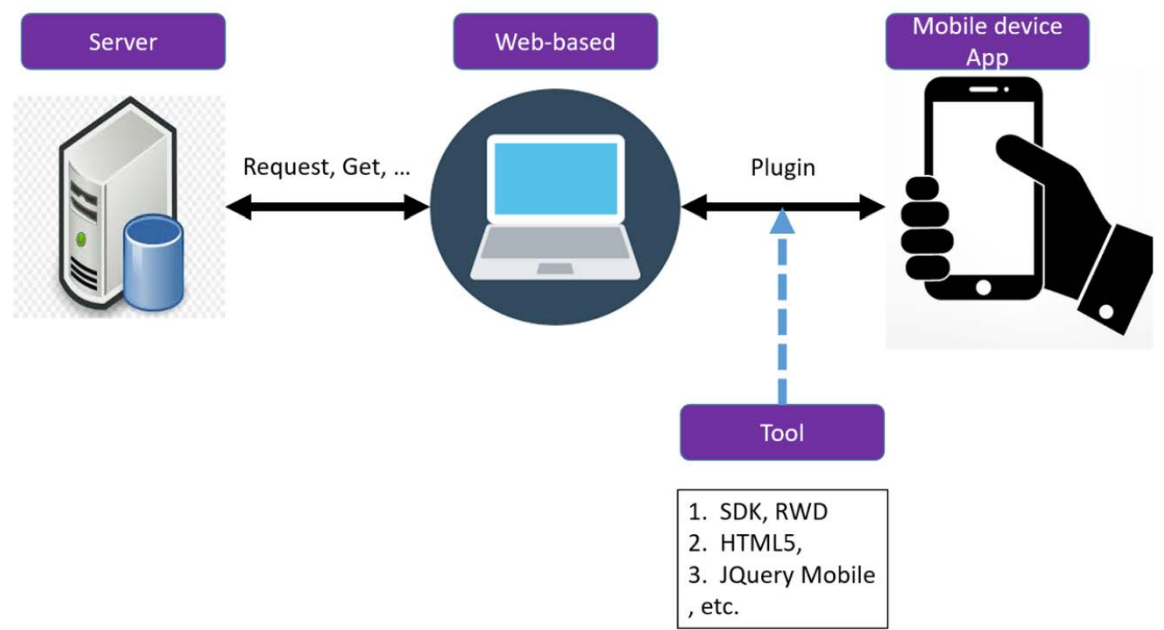

Figure 4. Mobile communication device integration webpage. 


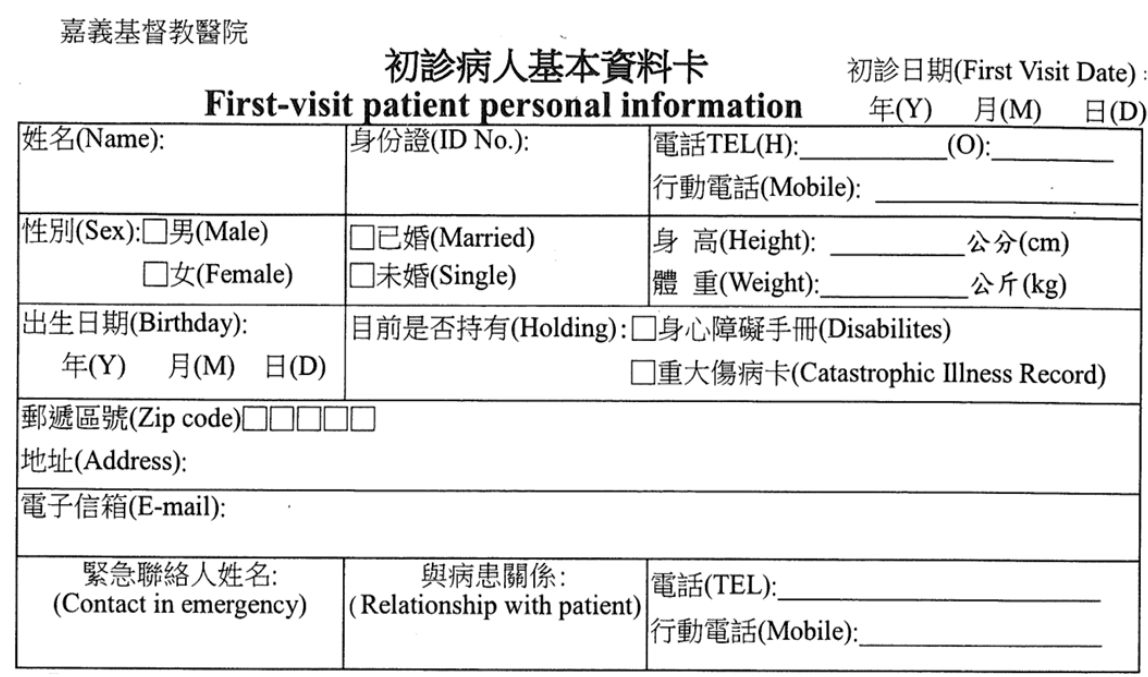

Figure 5. Example of a patient personal information.

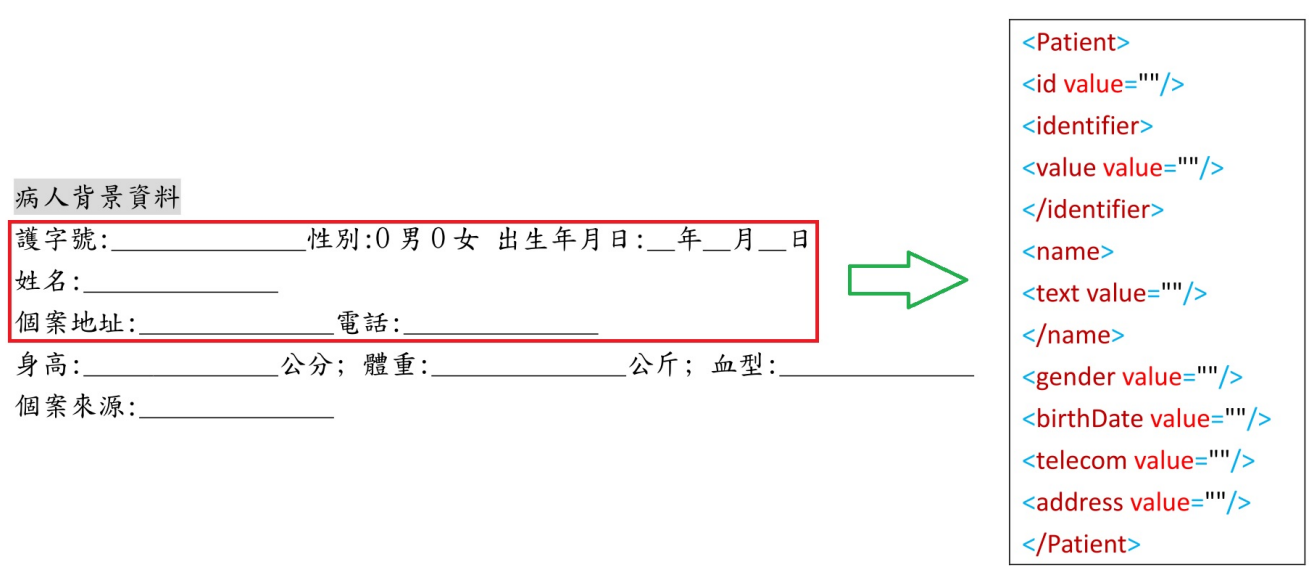

Figure 6. FHIR corresponding field.

of Chronic condition is also increasing year by year. Usually, the elderly with Chronic condition need to go back to the hospital for treatment, but for the elderly, it is a great physical burden. Therefore, through the telemedicine platform system we have developed, the patient and the medical end conduct medical consultation, as shown in Figure 7, and automatically store the medical data record, and use the collection of medical data as the data analysis data of the telemedicine system.

In this telemedicine system platform, the system will automatically collect various medical data of patients. Through the medical record data collected in the HL7 FHIR format, various patient physiological data, and medical history data are stored. The system can according to different data such as blood pressure, weight, a heartbeat before the physiological data has deteriorated, a reminder is issued in advance to alert patients to precautions and related measures. In addition, doctors use platform analysis to understand the user's use of the platform and the characteristics of the user population. Provide the improvement direction of this system and understand the user experience. 


\subsection{Telemedicine Login System Design}

According to the user's authority, we are divided into two identities: medical staff and patient. Figure 8 shows that the user chooses the login identity according to his own authority when logging in to the system.

\subsection{Medical Information Query}

The user can select the medical data of the patient according to the name and time of the visit. It is worth noting that the user is not limited to the medical staff or the patient himself, but in the part of querying the medical data, we select according to the user's login identity the difference is that if you are medical staff, you can query all patient data, but if you log in as a general user, you can only query your own medical information, as shown in Figure 9.

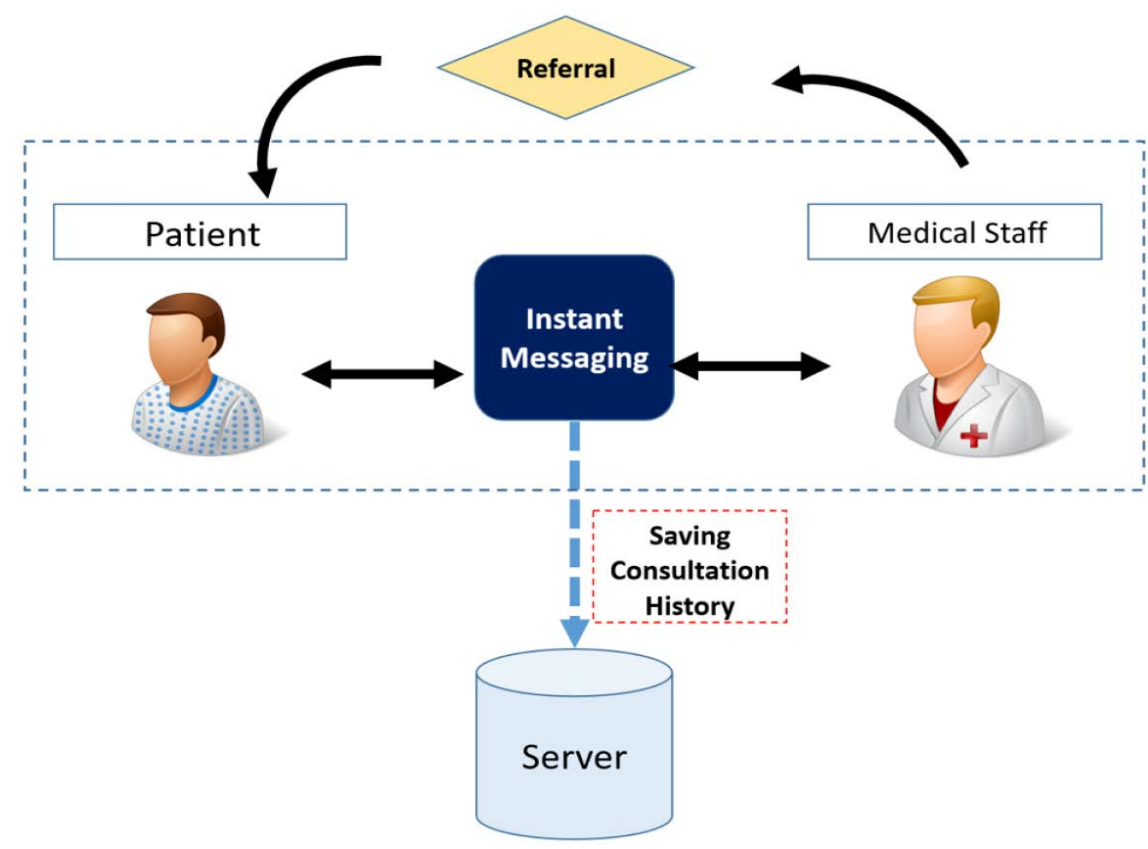

Figure 7. Telemedicine consultation process.

\section{WebApp Sign up Sign in}

\section{Member Login}

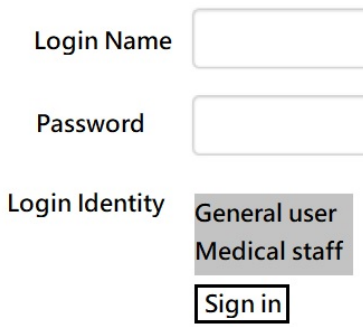

Figure 8. Telemedicine login system. 


\section{Danny Lin}

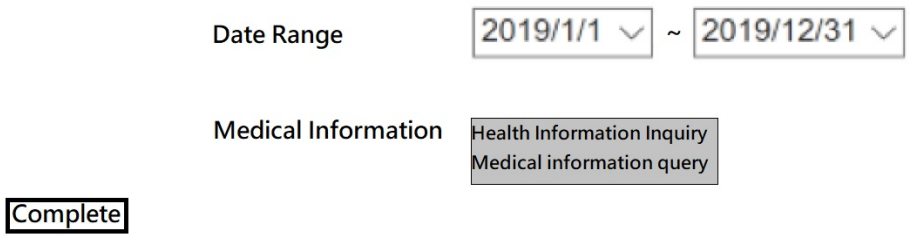

Figure 9. Patient information query.

\section{Conclusion}

We proposed a rapid and convenient telemedicine system and introduced the system to the Chiayi Christian Hospital. We received feedback from the hospital and found that most patients did not reject this system. Patients can use this system to avoid exhaustion when visiting a doctor, and our integration of the system into the mobile communication device, so that users can receive complete medical care without being restricted by equipment. The operation of this system meets the requirements of the medical consultation process. The reason is that the doctor analyzes the collected physiological data and medical record data. The medical staff can remind patients to pay attention to their physical conditions through the analysis results. However, a small number of patients feel that the system interface is not friendly enough. This is where we need to improve in the future.

\section{Acknowledgements}

We want to thank the budget supported by the Ministry of Education, Taiwan, under the grant of project Higher Education Sprout 110QA-2BA and number of 01113-01(National Chung Cheng University).

We want to thank the budget supported by Ditmanson Medical Foundation Chia-Yi Christian Hospital and the Center for Innovative Research on Aging Society (CIRAS) from The Featured Areas Research Center Program within the framework of the Higher Education Sprout Project by the Ministry of Education (MOE) in Taiwan.

\section{Conflicts of Interest}

The authors declare no conflicts of interest regarding the publication of this paper.

\section{References}

[1] Werner, M. (2009) Proceedings of the International Conference on Ageing. Wiener Klinische Wochenschrift, Beijing, 29-31 October 2008, 7-8.

[2] Fent, T. (2008) Department of Economic and Social Affairs, Population Division, United Nations Expert Group Meeting on Social and Economic Implications of Changing Population Age Structures. European Journal of Population/Revue Européenne de Démographie, 24, 451-452. https://doi.org/10.1007/s10680-008-9165-7 
[3] Koichi, Y., Fujiogi, M. and Koutsogiannaki, S. (2020) COVID-19 Pathophysiology: A Review. Clinical Immunology, 215, Article ID: 108427. https://doi.org/10.1016/j.clim.2020.108427

[4] Maia, M.R., Castela, E., Pires, A. and Lapão, L.V. (2019) How to Develop a Sustainable Telemedicine Service? A Pediatric Telecardiology Service 20 Years on-An Exploratory Study. BMC Health Services Research, 19, Article No. 681. https://doi.org/10.1186/s12913-019-4511-5

[5] Westlund, K., Attvall, S., Nilsson, R. and Jensen, O.C. (2016) Telemedical Maritime Assistance Service (TMAS) to Swedish Merchant and Passengers Ships 1997-2012. International Maritime Health, 67, 24-30. https://doi.org/10.5603/IMH.2016.0006

[6] World Health Organization (2010) Telemedicine: Opportunities and Developments in Member States. Report on the Second Global Survey on eHealth. 1st Edition, CAB Direct Ltd., Geneva.

[7] Ticia, G., Olazabal, V., Brown, K. and Pablos-Mendez, A. (2010) An Agenda for Action on Global E-Health. Health Affairs, 29, 233-236. https://doi.org/10.1377/hlthaff.2009.0934

[8] Pham, Q.-V., Fang, F., Ha, V.N., Jalil Piran, Md., Le, M., Le, L.B., et al. (2020) A Survey of Multi-Access Edge Computing in 5G and Beyond: Fundamentals, Technology Integration, and State-of-the-Art. IEEE Access, 8, 116974-117017. https://doi.org/10.1109/ACCESS.2020.3001277

[9] Chung, M.-A. and Chang, W.-H. (2020) Low-Cost, Low-profile and Miniaturized Single-Plane Antenna Design for an Internet of Thing Device Applications Operating in 5G, 4G, V2X, DSRC, WiFi 6 Band, WLAN, and WiMAX Communication Systems. Microwave and Optical Technology Letters, 62, 1765-1773. https://doi.org/10.1002/mop.32229

[10] Dolin, R.H., Alschuler, L., Beebe, C., Biron, P.V., Lee Boyer, S., Essin, D., et al. (2001) The HL7 Clinical Document Architecture. Journal of the American Medical Informatics Association, 8, 552-569. https://doi.org/10.1136/jamia.2001.0080552

[11] Lu, X., Gu, Y., Yang, L., Jia, W. and Wang, L. (2010) Research and Implementation of Transmitting and Interchanging Medical Information Based on HL7. The 2 nd International Conference on Information Science and Engineering, Hangzhou, 4-6 December 2010, 457-460. https://doi.org/10.1109/ICISE.2010.5689687

[12] Boussadi, A. and Zapletal, E. (2017) A Fast Healthcare Interoperability Resources (FHIR) Layer Implemented over i2b2. BMC Medical Informatics and Decision Making, 17, Article No. 120. https://doi.org/10.1186/s12911-017-0513-6

[13] Braunstein, M.L. (2018) Healthcare in the Age of Interoperability: The Promise of Fast Healthcare Interoperability Resources. IEEE Pulse, 9, 24-27.

https://doi.org/10.1109/MPUL.2018.2869317

[14] Prud'hommeaux Josh, E., Collins, J., Booth, D., Peterson, K.J., Solbrig, H.R. and Jiang, G. (2021) Development of a FHIR RDF Data Transformation and Validation Framework and its Evaluation. Journal of Biomedical Informatics, 117, Article ID: 103755. https://doi.org/10.1016/j.jbi.2021.103755

[15] Hoffman, R.A., Wu, H., Venugopalan, J., Braun, P. and Wang, M.D. (2018) Intelligent Mortality Reporting with FHIR. IEEE Journal of Biomedical and Health Informatics, 22, 1583-1588. https://doi.org/10.1109/JBHI.2017.2780891

[16] Bloomfield Jr., R.A., Polo-Wood, F., Mandel, J.C. and Mandl, K.D. (2017) Opening the Duke Electronic Health Record to Apps: Implementing SMART on FHIR. International Journal of Medical Informatics, 99, 1-10. https://doi.org/10.1016/j.ijmedinf.2016.12.005 


\section{Appendix A. Organization Structure of FHIR}

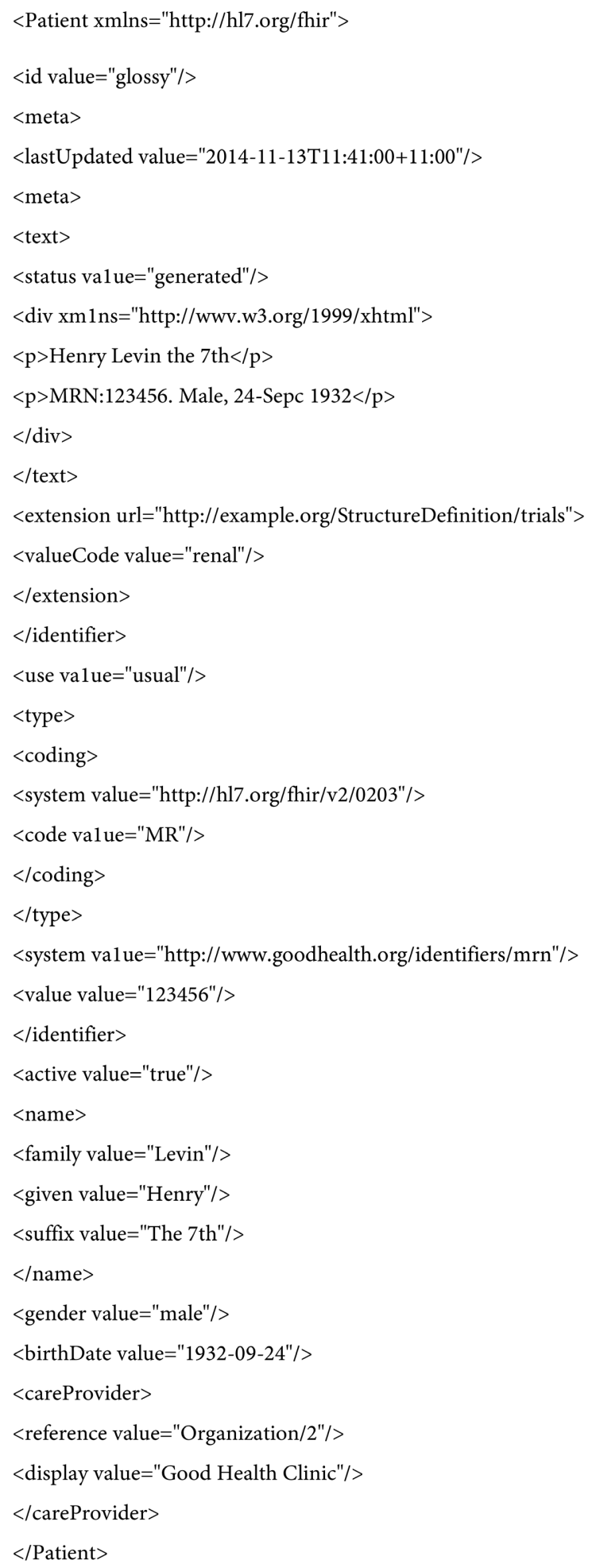




\section{Appendix B. Telemedicine System Development Environment}

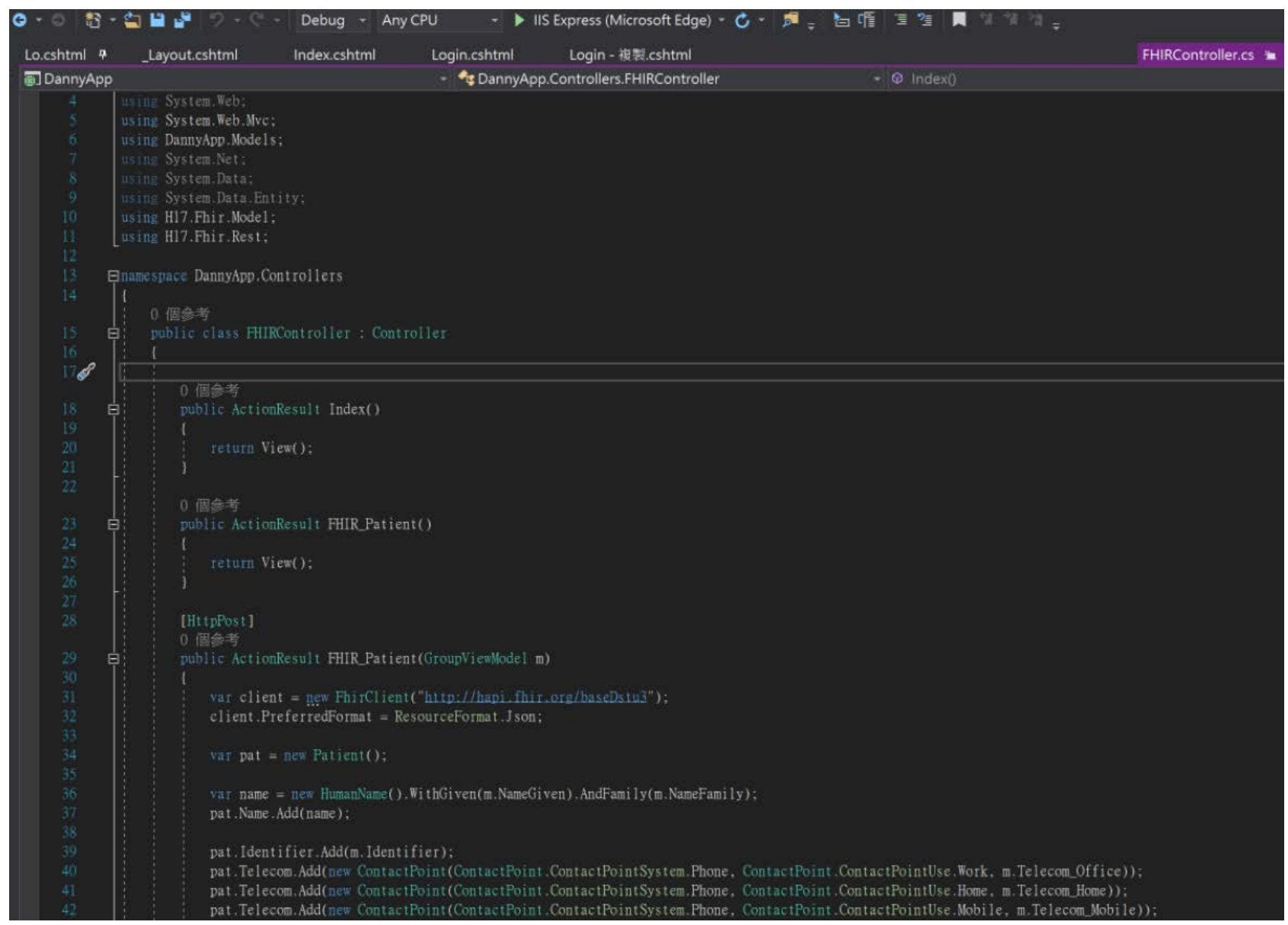

